\title{
Rafael MANDRESSI, dir., « Figures de la preuve ».
}

Communications, 84

Paris, Éd. Le Seuil, 2009, 171 p.

Nathalie Nadaud-Albertini

\section{CpenEdition}

\section{Journals}

Édition électronique

URL : http://journals.openedition.org/questionsdecommunication/261

DOI : 10.4000/questionsdecommunication.261

ISSN : 2259-8901

Éditeur

Presses universitaires de Lorraine

Édition imprimée

Date de publication : 30 juin 2010

ISBN : 978-2-8143-0024-8

ISSN : $1633-5961$

\section{Référence électronique}

Nathalie Nadaud-Albertini, «Rafael MANDRESSI, dir., "Figures de la preuve ». Communications, 84 », Questions de communication [En ligne], 17 | 2010, mis en ligne le 23 janvier 2012, consulté le 22 septembre 2020. URL : http://journals.openedition.org/questionsdecommunication/261 ; DOI : https://doi.org/10.4000/questionsdecommunication.261

Ce document a été généré automatiquement le 22 septembre 2020.

Tous droits réservés 


\section{Rafael MANDRESSI, dir., « Figures de la preuve ». Communications, 84}

Paris, Éd. Le Seuil, 2009, 171 p.

Nathalie Nadaud-Albertini

\section{RÉFÉRENCE}

Rafael MANDRESSI, dir., « Figures de la preuve ». Communications, 84. Paris, Éd. Le Seuil, $2009,171 \mathrm{p}$.

1 Cette livraison de Communications travaille la thématique de la preuve selon deux axes : l'acte de prouver et les disciplines où elle est fondamentale. Identifiant les lignes de force de la preuve, elle établit moins un état de lieux de recherche sur la preuve qu'il n'esquisse les contours d'un programme de recherche à prolonger. La diversité des domaines abordés en fait l'intérêt et la richesse. C'est pourquoi nous en présenterons la teneur sans l'amputer de cette variété foisonnante en proposant un aperçu de chaque article. Ce qui permet au lecteur de prendre la mesure du kaléidoscope de la preuve que constitue sa valeur.

Emmanuelle Danblon questionne un paradoxe de la rhétorique : l'opposition entre la raison comme garantie de validité et les émotions comme facteur de persuasion. L'auteure en interroge la source grâce à l'indice nécessaire d'Aristote, puis grâce au raisonnement indiciaire. Le premier se reconstruit par la déduction et conduit à une conclusion logique mais sans apport d'information. Le second repose sur l'abduction qui, dans un monde clos, ne permet qu'une unique hypothèse. L'apparition d'une alternative signe le passage de la pensée mythique à la pensée scientifique et condamne le raisonnement indiciaire comme irrationnel. On ampute alors la rationalité de sa racine : si une preuve doit subir une phase de justification, les conjectures antérieures sont nécessaires, sinon il n'y aurait rien à examiner. Le paradoxe se résout en dissociant découverte et justification. La première fournit des hypothèses. La seconde évalue les conjectures, introduisant le critère épistémologique de la validité. 
3 Les tensions entre la rationalisation croissante de la preuve et la liberté du juge dans l'établissement de la vérité judiciaire sont traitées par Jean-Louis Halpérin qui distingue deux moments. Le premier court $d u X X^{e}$ au XIX ${ }^{e}$ siècle. Une ligne prédomine : parole, écrit, libre-arbitre du juge et intime conviction. Jurés et juges ont donc une grande marge de liberté d'appréciation. Les dernières décennies ont été marquées par la prééminence de la preuve scientifique grâce à l'utilisation de l'ADN et des enregistrements de sons et d'images apparaissant comme incontestables. Ce mode de preuve affecte particulièrement le droit de la filiation et le droit pénal, même s'il demeure tempéré par l'appréciation du juge et/ou des jurés. La vérité judiciaire s'établit donc toujours "dans les meilleures conditions de sincérité et de publicité possibles » (p. 31), s'appuyant sur la liberté du juge d'évaluer les faits pour dire le droit. Le recours à la technique se retrouve dans la contribution de Claude Rosental qui s'intéresse à l'élaboration d'un logiciel d'astronomie et d'astrophysique pointant une tension fondamentale: l'obligation de réaliser des démonstrations pour justifier l'utilité de la recherche et le travail quotidien de laboratoire, et ce sur plusieurs facteurs. Le premier concerne l'adaptation du logiciel en fonction du domaine de calcul : son apparente transposabilité universelle occultait l'ajustement aux problèmes traités. Le second est l'exemplification : une série d'expériences a obligé à étudier la différence entre la démarche probatoire humaine et celle du logiciel. Le troisième touche à la rapidité de traitement lors des démonstrations, conduisant à sélectionner des outils produisant des statistiques favorables. Le quatrième concerne la dextérité de l'utilisateur dont le travail de formulation graphique en fait un acteur à part entière. D'où la nécessité de fabriquer un périphérique adapté aux utilisateurs amateurs.

4 En contrepoint, la preuve anthropologique est abordée par Mondher Kilani à travers le cannibalisme. L'auteur pointe le processus de codage grâce auquel les indices, traces et témoignages sont constitués en preuves. Il s'appuie sur deux exemples : le kuru et le cannibalisme préhistorique. Le kuru est une maladie du système nerveux, longtemps attribuée au cannibalisme qu'aucune preuve directe n'appuyait. Cette hypothèse provenait de l'utilisation d'une catégorie de pensée (l'indigène cannibale) légitimée par les discours anthropologiques et médicaux. Dans le second exemple, la recherche de preuves concerne les indices et les traces provenant de fouilles. Ce terrain d'investigation est une référence incontournable du discours anthropologique qui fait du cannibalisme préhistorique un lieu commun de la vulgate scientifique, alors que d'autres hypothèses sont possibles pour expliquer indices et traces. In fine, c'est le réservoir de récits disponibles qui fait basculer l'interprétation de la preuve dans le sens du cannibalisme.

5 Deux articles s'ancrent dans le domaine des mathématiques. Jean Dhombres s'attaque à « la preuve mathématique en tant qu'elle est preuve de mémoire » (p. 59); il commence par définir la preuve : un discours complet sur l'adéquation à ce qu'il prouve présentant de fait une dimension interprétative. Puis, il pointe le fait qu'un foisonnement de preuves permet de conclure au théorème de Pythagore. Conserver cette multitude de preuves permet de conserver la mémoire qu'elles contiennent. Ensuite, il s'intéresse au calcul algébrique qui se prouve par lui-même et réfère au Discours de la Méthode comme «métaphore d'un savoir bien mené »(p. 76), expliquant que, parfois, Descartes procède sans preuve réellement convaincante. Enfin, à travers la chaîne de travaux et d'attributions d'auctorialité du "théorème fondamentale de l'algèbre» (p.77), il montre combien une impression fausse pèse sur l'inconscient collectif des 
mathématiques au point de transformer la mémoire en preuve. À partir d'un exemple tiré des mathématiques de la Chine ancienne, Karine Chemla montre que reconnaître l'écriture d'une démonstration ne va pas de soi. Elle pointe que l'identification des textes demande un travail interprétatif reposant sur les pratiques de la culture épistémologique où le texte a été écrit. En effet, les textes s'accompagnent de commentaires fondamentaux pour leur pleine compréhension. Il convient alors d'adopter cette méthode de recherche : «Identifier les éléments pertinents [permettant de] localiser, dans les sources, les témoignages susceptibles de nous éclairer sur les pratiques dont ils [les textes] ont fait l'objet » (p. 98).

6 De son côté, Rafael Mandressi examine la preuve par les sens dans les sciences du corps. À partir du XVI ${ }^{e}$ siècle, seules les connaissances sensorielles sont considérées comme des preuves fiables de l'anatomie. Sont organisées des dissections publiques où démontrer revient à montrer le savoir et les voies de connaissance. Une question se pose : comment répondre aux exigences de la preuve sensorielle sans cadavre? On utilise alors l'image imprimée certifiée par l'œil de l'anatomiste et divers commentaires textuels ou par différentes stratégies d'écriture. Dans les écrits, les témoignages sont fondamentaux, qu'ils proviennent de témoins immédiats ou virtuels (les lecteurs des textes scientifiques). L'écriture suit le déroulement de la dissection et cite des personnes illustres comme témoins. Les figures des preuves sensorielles furent prolongées au XVII ${ }^{e}$ siècle.

7 La preuve a aussi à voir avec des dimensions irrationnelles. Ainsi la preuve rumorale est-elle l'objet du travail de Bernard Paillard qui pointe l'importance du témoignage comme modalité de certification. La nature des relations avec le témoin est fondamentale : on croit et on propage plus facilement un «on-dit » quand il émane d'un proche, d'une personne dont remettre en doute la parole reviendrait à rompre le rapport de confiance, de proximité, de sympathie, de connivence idéologique ou d'autorité. Le démenti ne sert pas de preuve contrant la rumeur, car il émane d'instances institutionnelles qui suscitent la méfiance. La rumeur se développe en situation d'incertitude, ouvrant un réservoir de schèmes de croyance tels que le savant fou, le complot, la puissance de l'argent, le réseau de notables, le sida, la pédophilie, etc. Elle dispose également d'une argumentation propre: sans fait probant, toutes les modalités de raisonnement sont mobilisées, entrelaçant indices, hypothèses, déductions, induction, analogies, tautologie, etc. La rumeur est alors un système d'idées négligeant l'épreuve du réel. Quant à Jacqueline Carroy, elle travaille sur la preuve onirique à travers une controverse qui a ébranlé l'autorité scientifique d'Alfred Amaury qui, au XIX ${ }^{e}$ siècle, apparaissait comme le fondateur d'une science des rêves. Il prônait une méthode d'observation objective consistant à noter ses rêves au réveil, à les collecter et à les archiver. Un rêve spécifique fit débat. Jacques Le Lorrain l'envisagea comme une œuvre de l'imagination. Frédéric Paulhan insista sur la reformation du rêve dans le souvenir. Paul Tannery le considéra comme une succession de tableaux recomposés en récit au réveil. Victor Egger proposa une narration scientifique des rêves consistant à les décomposer et non à les retranscrire en récit. Quant à Marcel Foucault, considérant que les rêves sont toujours remémorés, il envisagea une méthode indirecte à travers la notation directe et indirecte, et la transcription télégraphique. Cette controverse a marqué Freud qui considère les rêves comme "des récits trompeurs dont il faut décomposer l'apparente cohérence pour en déchiffrer les éléments latents, à la manière d'un rébus » (p. 146). 
La réflexion se clôt sur deux contributions relatives à l'histoire. Christine Jungen s'intéresse à la preuve en matière d'archives historiques. Elle travaille sur les pratiques du Centre de documents et manuscrits de l'université jordanienne qui construit un fonds d'archives accessibles et maniables par les historiens. Elle montre comment l'archive est fabriquée. Le document administratif original est transformé en archive au cours d'un processus spécifique. Il mobilise des opérations de manipulation (tri, classement, rangement) et des procédures techniques (confection de pochette en coton, mesure de l'humidité). Et, il reproduit et démultiplie l'original : il est tout d'abord extrait sous forme de copie (microfilms ou photocopies), puis transcrit dans un ordre textuel accroissant son exploitabilité. Reproduction comme réécriture limitent le recours à l'original et surtout font des formes reproduites des substituts valides renversant le rapport entre original et copie. En effet, seule l'archive fabriquée est probatoire. Pour sa part, Nicole Lapierre interroge la preuve vidée de toute dimension humaine. Elle étudie la démarche d'un historien « amateur ", Jean-Claude Pressac, dont les travaux ont connu un succès non négligeable. Il a travaillé sur l'ensemble du dispositif technique ayant permis l'extermination des Juifs pendant la Seconde Guerre mondiale. Négationniste dans un premier temps, les documents recueillis à Auschwitz le convainquent jusqu'à le transformer en farouche contempteur du négationnisme. De façon froide et dépassionnée, il traite la question des possibilités techniques de la Shoah, preuves écrites, mesurables, logiques à l'appui. Il exclut donc de sa démarche tout témoignage, considérant que la mémoire humaine n'est pas fiable. Bien que montrant la force de la preuve, Jean-Claude Pressac incarne également la question d'une science sans conscience, se situant dans le domaine de la technique pure, négligeant toute dimension humaine. Sa démarche est alors à considérer comme « une vue limitée par rapport à une démarche historique, qui dans sa visée, doit inclure la vérité prouvée et la vérité éprouvée » (p.171). En définitive, cette livraison sur les « figures de la preuve » pose les jalons d'une histoire de celle-ci qui reste à construire.

\section{AUTEURS}

\section{NATHALIE NADAUD-ALBERTINI}

CEMS, École des hautes études en sciences sociales

nnadaud@noos.fr 